\title{
TESTES DE TOXICIDADE COM EMBRIÕES DE Perna perna (LINNAEUS, 1758) (MOLLUSCA: MYTILIDAE): AVALIAÇÕES DE INDUÇÃO E SENSIBILIDADE
}

\author{
REIS Fo., R.W. \& C. RESGALLA Jr. \\ CTTMAR/UNIVALI - Cx.P. 360, Itajaí, SC - 88.302-202 \\ rireis@mailcity.com - resgalla@cttmar.univali.br
}

RESUMO

\begin{abstract}
O objetivo principal deste trabalho foi realizar experimentos que fornecessem uma base para elaboração de técnicas adequadas ao desenvolvimento de testes de toxicidade embriolarvais com o mexilhão nativo Perna perna. Assim organismos adultos foram investigados quanto a técnica mais apropriada e eficaz para liberação de gametas e que resultasse em gametas de boa qualidade. Os embriões foram submetidos a variações de temperatura, salinidade e tempo de incubação, determinando o fim de teste ("endpoint") e os parâmetros ideais para a realização dos testes de toxicidade. Foi executado baterias de testes utilizando a substância de referência Dodecil Sulfato de Sódio (DSS), com tratamento dos dados para a estimação da Concentração de Efetiva ( $\mathrm{CE}_{50}$ - aguda) e Concentração de Efeito Não Observado (CENO - crônico). Os valores de $\mathrm{CE}_{50}$ para o DSS oscilaram entre $0,45\left(20^{\circ} \mathrm{C} / 48 \mathrm{~h}\right)$ e $1,88 \mathrm{mg} / \mathrm{l}\left(25^{\circ} \mathrm{C} / 24 \mathrm{~h}\right)$ e o CENO de $0,185 \mathrm{mg} /$ I $\left(20^{\circ} \mathrm{C} / 48 \mathrm{~h}\right)$ a $0,75 \mathrm{mg} / \mathrm{l}\left(25^{\circ} \mathrm{C} / 24 \mathrm{~h}\right)$. Comparando-se os valores obtidos com os resultados encontrados para outros organismos testes, utilizando a mesma substância de referência, constatou-se que embriões de Perna perna apresentam uma alta sensibilidade, onde os tratamentos crônicos resultam em valores de efeito mais reprodutíveis.
\end{abstract}

Palavras chave: Teste Embrio-Larval; Mexilhão; Perna perna; DSS; Liberação de Gametas.

\section{ABSTRAT \\ TOXICITY TESTS WITH EMBRYOS OF MUSSEL Perna perna: EVALUATION OF SPAWNING AND SENSIBILITY}

\begin{abstract}
The main goal of this paper was to set up guidelines to standartize the procedure of developing embrio-larval toxicity tests using the native Brazilian mussel Perna perna (Mollusca: Mytilidae). In vitro spawning tests were carried out using adult organisms in order to obtain healthy gametes. Embryos development response caused by variation of temperature, salinity and incubation time was investigated in order to determine the end point and ideal parameter settings to develop toxicity assays. Different data sets treatments were carried out using the reference chemical Dodecyl Sodium Sulfate (DSS) in acute (Effective Concentration - $\mathrm{EC}_{50}$ ) and chronic (No-ObservedEffect Concentration - NOEC) tests. The $\mathrm{EC}_{50}$ for DSS ranged from $0,45\left(20^{\circ} \mathrm{C} / 48 \mathrm{~h}\right)$ to $1,88 \mathrm{mg} / \mathrm{l}$ $\left(25^{\circ} \mathrm{C} / 24 \mathrm{~h}\right)$, while NOEC values ranged from $0,185 \mathrm{mg} / \mathrm{l}\left(20^{\circ} \mathrm{C} / 48 \mathrm{~h}\right)$ to $0,75 \mathrm{mg} / \mathrm{l}\left(25^{\circ} \mathrm{C} / 24 \mathrm{~h}\right)$. Comparing the Perna perna embrios response with those from other test organisms, it was observed that this species exhibited a high sensibility, where a more accurate response were found in chronic treatments.
\end{abstract}

Key Words: Embryo-Larval Tests; Mussel; Perna perna; DSS; Spawning Induction.

\section{INTRODUÇÃO}

Os testes de toxicidade aquática são uma técnica eficaz para avaliar o grau de con- taminação ambiental, bem como a toxicidade de efluentes que são lançadas nos ambientes; permitindo o estabelecimento de limites permissíveis de liberação de vários agentes quí- 
micos de acordo com as normas estabelecidas pelo Conselho Nacional do Meio Ambiente (CONAMA).

O emprego de organismos marinhos em testes de toxicidade é, de certa forma, recente, tendo uma grande expansão somente nos últimos anos (Reishi, 1988). Com a defasagem científica nos países em desenvolvimento, a grande maioria de testes padronizados e protocolados são referentes a espécies alóctonas, existindo uma carência no que diz respeito a adequação de espécies nativas (fauna e flora) ao desenvolvimento de testes de toxicidade. No Brasil, os últimos encontros científicos sobre o tema têm apontado os organismos mais utilizados em testes crônicos e agudos como: algas (Amado Filho et al., 1997 e Carvalheira et al., 1997); misidáceos (Zamboni et al., 1996); copépodas (Nipper et al., 1993 e Resgalla Jr. et al., 1997); equinodermos (Badaró-Pedroso \& Santos, 1996a; BadaróPedroso et al., 1998); decápodas (BadaróPedroso \& Santos, 1996b; Lucero et al., 1997 e Moraes et al., 1996); e peixes (Resgalla Jr. et al., 1998 e Sampaio \& Tesser, 1997 e Moraes et al., 1996).

Testes de toxicidade com invertebrados aquáticos fornecem um importante suporte na determinação de impactos químicos impostos ao meio ambiente, principalmente devido ao seu ciclo de vida rápido e à alta sensibilidade apresentada nos primeiros estágios de desenvolvimento (Rand \& Petrocelli, 1985).

As larvas de moluscos bivalves possuem grande representatividade na comunidade marinha planctônica ao redor do mundo (Hooker, 1997). No hemisfério Norte são bastante investigadas, tanto quanto ao seu desenvolvimento, como às respostas apresentadas em testes de toxicidade, destacando-se os trabalhos elaborados por Bayne (1965); Bayne et al. (1975); Cherr et al. (1990); Davis (1961); Fichet et al. (1998); His \& Beiras (1995); e Hunt \& Anderson (1993).

No Brasil, o mexilhão Perna perna possui grande potencial de aplicabilidade para ser utilizado em testes de toxicidade, pois preen- che vários requisitos (Greenberg et al., 1992) para ser selecionado como espécie bioindicadora: sendo de fácil coleta; disponibilidade ao longo do ano; adaptabilidade a condições laboratoriais; sensibilidade aos compostos químicos; importância econômica e ecológica comprovada.

O mexilhão Perna perna é morfologicamente muito semelhante a espécie Mytilus edulis encontrada na América do Norte e que possui testes padronizados pela EPA (1991) e ASTM (1992)

Taxonomicamente obedece a seguinte classificação, segundo Rios (1994)

\section{Phylum: MOLLUSCA \\ Classe: BIVALVIA}

Sub-ordem: Pteriomorpha

Ordem: Mytiloidea (Férussac, 1822)

Superfamília: Mytilacea (Rafinesque, 1815)

Família: Mytilidae (Rafinesque, 1815)

Subfamília: Mytilinae (Rafinesque, 1815)

Espécie: Perna perna (Linnaeus, 1758)

Possui ampla distribuição latitudinal, ocorrendo do Norte do Espírito Santo até o Sul do Rio Grande do Sul (Fernandes, 1981), habitando a região entre-marés do supra-litoral inferior até a profundidade de 19 metros, fixo a rochas ou qualquer outra estrutura rígida imersa.

A fase embrio-larval do Perna perna faz parte do plâncton, por cerca de 50 dias, até sua fixação, sendo assim meroplanctônica. Aproximadamente 6 horas após a fecundação, são formadas as larvas trocóforas, que depois de 24 horas passa a véliger (larva D), seguida pela fase de veliconcha e após decorridos \pm 40 dias, pedivéliger, quando possuem fototropismo negativo e geotropismo positivo (Romero, 1980).

O presente estudo, visa levantar subsídios que contribuam para que a espécie nativa de mexilhão tenha sua aplicação em testes de toxicidade embrio-larvais normatizada, pois os estudos enfocando esta área são escassos, envolvendo esparsas referências (Abessa et al., 1998; Jorge \& Moreira, 1998) e inexistência de trabalhos publicados. 


\section{METODOLOGIA}

Adultos de Perna perna, apresentando em média $50 \mathrm{~mm}$ de comprimento, foram coletados na estação experimental de maricultura (Campus V - UNIVALI), localizado no município de Penha, litoral Norte do Estado de Santa Catarina ( $26^{\circ} 48^{\prime} S 48^{\circ} 36^{\prime}$ W). Após a coleta, os mexilhões foram transportados ao laboratório, onde foram limpos e mantidos em solução de hipoclorito de sódio (comercial na diluição de 1:1000) por 30 minutos, para limpeza externa evitando contaminação com outros organismos.

\section{Indução e Coleta do Material Gâmico}

Como mexilhões são animais de fertilização externa, o sucesso de liberação de gametas foi instigada segundo diferentes técnicas apresentadas por Bayne (1976):

1 - Choque Térmico: Inicialmente os organismos foram mantidos em frascos plásticos de 1 litro contendo $500 \mathrm{ml}$ de água do mar filtrada em incubadora $\left(25^{\circ} \mathrm{C}\right)$, transferidos a água refrigerada $\left(15^{\circ} \mathrm{C}\right)$ e subsequentemente a água aquecida $\left(32^{\circ} \mathrm{C}\right)$; sendo mantidos por cerca de 30 minutos em cada condição.

2 - Castigos: O termo castigo é definido como o intervalo de tempo em que os organismos são deixados fora da água. Foi executado o castigo em que os animais foram mantidos a seco por 24 horas antes dos testes.

3 - Utilização de substâncias Químicas: A utilização de substâncias no estímulo da desova em bivalves foi feita com a adição ao meio de cloreto de potássio $(\mathrm{KCl}) 1 / 100$ de $0,5 \mathrm{M}$, ou hidróxido de amônio $\left(\mathrm{NH}_{4} \mathrm{OH}\right) 1 / 100$ de $0,1 \mathrm{M}$ (Sagara, 1958) ou peróxido de hidrogênio $(1 \mathrm{ml} /$ I); ou ainda pela injeção de $2 \mathrm{ml}$ de $\mathrm{KCl} 0,5 \mathrm{M}$ (Iwata, 1951) ou $\mathrm{NH}_{4} \mathrm{OH} \mathrm{0,1M}$ (Sagara, 1958) na cavidade do manto.

4 - Indução Elétrica: O emprego de choque elé- trico (9 volts) para estimular a descarga reprodutiva seguiu o método de Iwata (1950).

5 - Extrato de Gônadas: Extratos de testículos macerados foram preparados e inoculados (2 $\mathrm{ml}$ ) nos frascos com os organismos adultos.

6 - Retirada do Bisso: O bisso (conjunto de fibras pelo qual o animal se fixa ao substrato) foi removido juntamente com a musculatura da glândula bissal.

7 - Fluxo Contínuo: Um sistema de fluxo contínuo de água do mar filtrada foi desenvolvido em forma de cascata, sendo os mexilhões colocados em placas de petri no fundo de calhas para que os gametas liberados não se perdessem (Zaroni, com.pess. - IOUSP).

Obtendo-se as liberações dos gametas, o esperma foi transferido através de pipeta de Pasteur para béquer de $50 \mathrm{ml}$ e mantido sobre gelo, os ovócitos para béquer de $400 \mathrm{ml}$ contendo água do mar filtrada na salinidade do teste.

\section{Procedimento para Fertilização}

Inicialmente os ovócitos foram filtrados em malhas de $140 \mu \mathrm{m}$ para remoção de impurezas. Foi inoculada a quantidade de $1 \mathrm{ml}$ de esperma em solução de $400 \mathrm{ml}$ de ovócitos, sendo misturados periodicamente por $20 \mathrm{mi}$ nutos através de leve agitação com bastão de vidro. A verificação do sucesso de fertilização (formação do corpúsculo polar) e também da densidade foi executada através de contagem em câmara de Sedgewick-Rafter sob aumento de 200 vezes. Após a fecundação, a água do mar da solução de ovos foi renovada três vezes pela retirada do sobrenadante após sua precipitação.

\section{Montagem dos Testes}

Em cada experimento desenvolvido neste estudo, utilizou-se frascos-teste de material 
plástico de $15 \mathrm{ml}$ contendo um volume de 10 $\mathrm{ml}$ de substância teste em 7 concentrações. Para cada teste foram preparadas 4 réplicas, mais os frascos controle. Além disto, manteve-se o controle dos parâmetros físico-quími$\cos$ ( $\mathrm{pH}$ e salinidade) para cada concentração da substância.

Em cada frasco teste foram adicionados $200 \mu l$ de solução de ovos, contendo um número mínimo de 300 ovos para cada frascoteste, com posterior cobertura dos frascos com filme plástico. Assim, os lotes foram levados à temperatura teste e mantidos com fotoperíodo (12 horas claro 12 horas escuro).

Os seguintes testes estáticos foram realizados :

1 - Testes de desenvolvimento em diferentes tempos de incubação 18, 24, 36, 48, e 72 horas, a temperatura de $20^{\circ} \mathrm{C}$.

2 - Testes de desenvolvimento em diferentes salinidades $15,20,25,30,35$ e $40 \%$, a temperatura de $20^{\circ} \mathrm{C}$.

3 - Testes com a substância de referência dodecil sulfato de sódio (DSS).

3.1 Testes de sensibilidade em duas temperaturas $\left(\mathrm{T}=20^{\circ} \mathrm{C} \mathrm{e} \mathrm{T}=25^{\circ} \mathrm{C}\right)$ nas concentrações de $6 \mathrm{mg} / \mathrm{l}$ até $0,185 \mathrm{mg} / \mathrm{l}$ com fator de diluição de 0,5 .

3.2 Testes em dois tempos de exposição (24 e 48 horas) nas concentrações de $6 \mathrm{mg} / \mathrm{l}$ até $0,185 \mathrm{mg} / \mathrm{l}$ a temperatura de $20^{\circ} \mathrm{C}$ com fator de diluição de 0,5 .

A salinidade da água de diluição usada nos testes, excetuando-se os testes de salinidade, foi em média de $33 \%$.

\section{Finalização dos Testes e Contagens}

Para a finalização de cada experimento, os frascos controle foram examinados quanto a dominância de larvas $\mathrm{D}$, com posterior fixação com $1 \mathrm{ml}$ de formol a $4 \%$ neutralizado. Para as contagens as amostras foram transferidas para câmara de Sedgewick-Rafter e analisadas em microscópio biológico, onde as categorias de ovo, larva trocófora, larva $D$ protraída (estado em que parte do manto do molusco encontra-se fora do abrigo da concha), larva $\mathrm{D}$, larva deformada e em decomposição (Fig. 1) foram registradas na quantidade mínima de 100 organismos conforme His \& Beiras (1995)

\section{Tratamento dos Dados}

A partir das contagens, foram calculadas as percentagens de efeito (PE) mediante a divisão entre o somatório das larvas trocóforas e deformadas sobre o número total de larvas (somatório de larvas trocóforas, deformadas e D protraídas e normais).

Para os tratamentos agudos, os dados brutos foram ajustados segundo o efeito observado no controle, quando foi o caso. O procedimento de análise para se obter os valores da Concentração Efetiva $\left(\mathrm{CE}_{50}\right)$ para cada teste seguiu o método indicado pela EPA (1991). A abordagem crônica na estimativa da Concentração de Efeito Não Observado (CENO) e da Concentração de Efeito Observado (CEO), utilizando-se análise de variância, também seguiram as recomendações da EPA (1991).

\section{RESULTADOS E DISCUSSÃO}

Técnicas aplicadas na indução de liberação de gametas

Na tabela 1 são apresentados os resultados obtidos nas diferentes técnicas utilizadas para estimular a liberação de espermatozóides e ovócitos.

Apesar da bilbliografia internacional relatar sucessos na liberação de gametas por bivalves através de várias das técnicas, o mexilhão nativo Perna perna mostrou-se refratário à maioria delas. Destaca-se que a técnica de fluxo contínuo com água do mar foi a única que na totalidade das tentativas houve reação positiva. Deve-se atentar que para um teste ser aceito segundo os critérios da ASTM (1992), o 
NOTAS TÉC. FACIMAR, 6: 7-17, 2002.

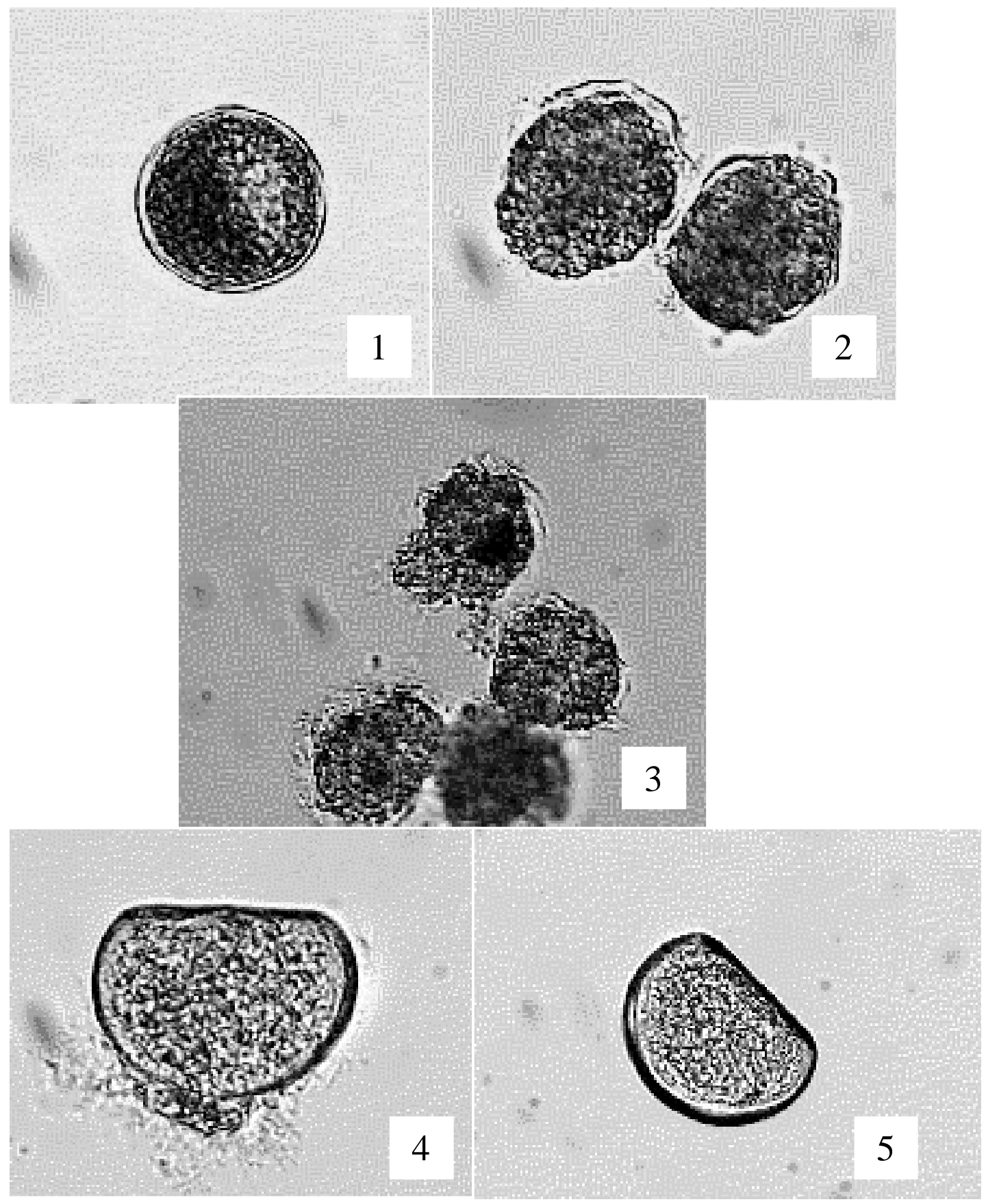

Figura 1: Microfotografias de larvas de Perna perna observadas no fim dos testes: 1- Ovo, 2 - Trocóforas, 3 Decomposição, 4 - D protraída e 5 - D normal. (200x) 
mínimo de $70 \%$ dos embriões introduzidos no controle devem resultar em larvas $D$ normais ao final do teste. Este fato reforça o emprego do sistema de fluxo contínuo como método indutor de desovas, pois este foi o único em que os PE nos controles ficaram dentro dos limites aceitáveis (média de $24 \%$ para os testes realizados), com um mínimo de $18 \%$ e um máximo de $35 \%$. Provavelmente a boa qualidade das desovas obtidas ao se aplicar o sistema de fluxo contínuo, seja um reflexo da menor agressividade do método, onde o fluxo contínuo da água do mar simula a dinâmica do ambiente para a dispersão dos gametas, ou ocasionando uma maior oxigenação da água.

Ressalta-se entretanto, que existe frequentemente a necessidade de conjugar diferentes técnicas de indução quando não é observada a desova em tempo hábil para a realização de testes de toxicidade. Assim, o uso de fluxo contínuo pode ser associado ao resfriamento da água, mas nunca o seu aquecimento, pois neste último caso, apesar de ocorrer a desova, os embriões não são viáveis para o desenvolvimento até larva D. Pode-se ainda empregar o uso alternado de Peróxido de Hidrogênio diluído e extratos de testículos adicionados à cavidade do manto e castigos entre séries de permanência em fluxo contínuo.

Ao se investigar o estágio de desenvolvimento (figura 2) o comportamento encontrado foi o esperado, ocorreu um decréscimo na quantidade de trocóforas, enquanto ocorria um acréscimo de larvas $D$ com o passar do tempo. $O$ desaparecimento de larvas $D$ anormais após decorridas 48 horas pode ser explicado pela decomposição e consequente sua fragmentação.

Um ponto a ser destacado é que a partir de 24 horas de incubação a forma $D$ é a dominante, permitindo assim a padronização da

Tabela 1: Técnicas utilizadas na indução de liberação de gametas, sucesso obtido e percentual de efeito observado no controle.

\begin{tabular}{lcc}
\hline Técnicas & Sucesso & Efeito no controle \\
\hline $3 \quad$ Choque Térmico & Positivo/Ocasional & $60 \%$ \\
$2 \quad$ Castigo $24 \mathrm{~h}$ & Positivo/Ocasional & $100 \%$ \\
A) Adição de Peróxido de & Positivo/Ocasional & $100 \%$ \\
Hidrogênio ao meio & & \\
B) Adição de $\mathrm{KCl}$ ao meio & Negativo & - \\
$4 \quad$ Injeção de $\mathrm{KCl}$ & Negativo & - \\
C) Injeção de $\mathrm{NH}_{4} \mathrm{OH}$ & Negativo & - \\
D) Adição de $\mathrm{NH}_{4} \mathrm{OH}$ ao meio & Negativo & - \\
E) Retirada do Bisso & Negativo & - \\
F) Choque elétrico & Negativo & - \\
G) Adição de extrato de gônadas ao & Negativo & \\
meio & & $24 \%$ (média) \\
12 Sistema de fluxo contínuo & Positivo/Frequente & \\
& (Decorridas $\approx 3$ horas) & \\
\hline
\end{tabular}


finalização dos testes (endpoint). Estudos realizados em outros laboratórios reportam a necessidade de 48 horas para a formação de larvas $D$ e consequente o seu domínio (Abessa, 1998). Tal fato provavelmente é inerente às populações empregadas, em função das condições ambientais em que se encontram. Esta diferenças populacionais podem ser destacadas pelo índice de condição (relação entre o peso dos tecidos e o peso total) entre os mexiIhões da região sudeste e sul do Brasil, onde no litoral de São Paulo o índice máximo observado é de 0,32 (Henriques, 2001) enquanto que em Santa Catarina este mesmo índice pode chegar a 0,47 (Wojciechowiski Jr., 1997).

His \& Beiras (1995) destacam o emprego de larvas $D$ protraídas como sendo organismos afetados em testes de toxicidade. Segundo a figura 3, é sugerido que este tipo de larva seria um estágio anterior as larvas $D$ normais diferenciando-se pelo crescimento da concha. Em 24 horas de teste, a porcentagem deste

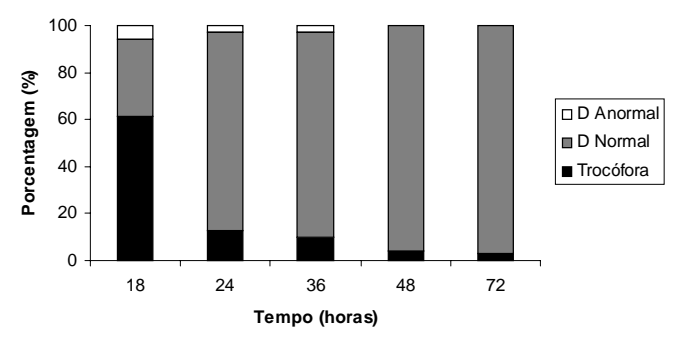

Figura 2: Estágios de desenvolvimento de larvas de Perna perna em função do tempo de incubação.

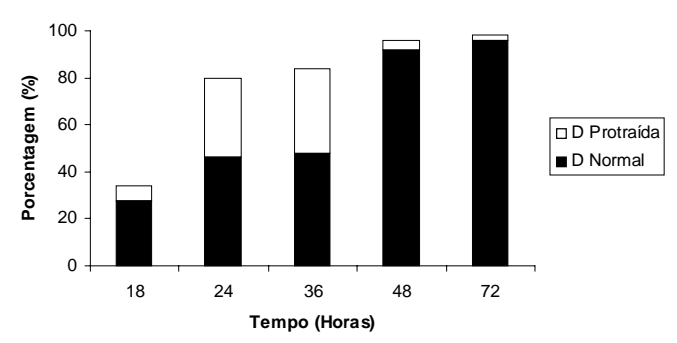

Figura 3: Estágios de D normal (DN) e D protraída (DP) em função do tempo de incubação. tipo de larva seria de $36 \%$ enquanto que em 48 horas o seu percentual foi de $6 \%$. Portanto, a finalização dos testes em 24 horas sem considerar este como critério de efeito, propicia a vantagem de se realizar testes mais curtos inclusive para situações que necessitem de avaliações emergenciais. Em 48 horas de teste o uso deste critério seria opcional, já que a identificação deste estágio nem sempre é possível de ser realizada com segurança.

\section{Testes de desenvolvimento a diferentes salinidades}

Observou-se pelos testes de desenvolvimento de larvas de Perna perna em diferentes salinidades (figura 4) que a faixa ideal de trabalho para a execução dos testes de toxicidade encontra-se entre 30 e $35 \%$, onde os extremos de salinidade interromperam o seu desenvolvimento.

\section{Testes com substância de referência - DSS}

A tabela 2 apresenta os resultados dos tratamentos crônicos e agudos obtidos para os testes com DDS em diferentes condições.

Comparando-se os testes de toxicidade executados a $20^{\circ} \mathrm{C}$ e a $25^{\circ} \mathrm{C}$, aparentemente observa-se uma maior sensibilidade apresentada pelos embriões à temperatura de $20^{\circ} \mathrm{C}$, tanto para o tratamento crônico (CENO) como para o agudo $\left(\mathrm{CE}_{50}\right)$. Entretanto, o esperado seria encontrar efeitos deletérios mais acentu-

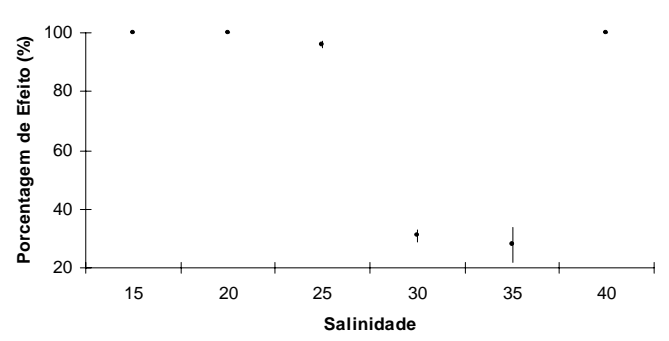

Figura 4: Média e Intervalo de Confiança de $95 \%$ da percentagem de efeito sobre larvas do Perna perna a diferentes salinidades 
ados em temperaturas maiores, pois esta normalmente atua potencializando os efeitos nocivos das substâncias e agindo de forma sinérgica (Rand \& Petrocelli, 1995). Porém uma maior percentagem de efeito (>30\%) registrada nos frascos-controle a $25^{\circ} \mathrm{C}$ sugere problemas de validação dos testes. Como os embriões foram provenientes do mesmo evento de desova e foram incubados em densidades iguais para ambas as temperaturas, o efeito maior nos frascos controle a $25^{\circ} \mathrm{C}$ deve ser interpretado como consequência de alterações metabólicas impostas pela temperatura mais elevada, não sendo, portanto, a temperatura recomendada para a execução dos testes de toxicidade.

Quanto ao tempo de exposição ao DSS por 24 e 48 horas, a análise aguda para 24 horas e 48 horas, apresentaram praticamente os mesmos resultados, não indicando a influência do tempo sobre os percentuais de efeito. Porém, a análise crônica revelou que a concentração de efeito observado para 48 horas é um fator de diluição menor do que a de 24 horas, sendo esta uma resposta esperada. Como a substância química testado se trata de um detergente, este age a nível de membrana celular, prejudicando assim as trocas entre o or- ganismo e o meio externo. Assim, quanto maior o tempo de exposição maiores serão os efeitos observados.

De forma geral, observou-se que dentro das mesmas condições o CENO manteve-se constante, enquanto que $\circ \mathrm{CE}_{50}$ apresentou uma maior variação, o que confirma a maior sensibilidade do tratamento agudo à manipulação dos dados em função do controle. Este fato sugere que, em casos onde os organismos-teste são muito sensíveis, a aplicação do tratamento crônico apresenta vantagens sobre o tratamento agudo.

Um ponto importante é comparar os valores de $\mathrm{CE}_{50}$ com DSS encontrados para Perna perna com resultados apresentados por outros autores e também para outros organismos. No trabalho de Abessa et al. (1998) também realizado com embriões de Perna perna foi obtido um $\mathrm{CE}_{50}$ de $1,31 \mathrm{mg} / \mathrm{l}$ ( $48 \mathrm{~h}$ de exposição), já nos testes realizados por Nipper et al. (1993) utilizando os copépodas Acartia lilljeborgie Temora stylifera foram encontrados $\mathrm{CE}_{50}$ de 2,6 a $1,4 \mathrm{mg} / \mathrm{l}$ e 3,00 a 2,1 mg/l respectivamente, e para o misidáceo Mysidopsis juniae o $\mathrm{CE}_{50}$ variou de 2,2 a 2,3 mg/l. Carr et al. (1996) relatam que para embriões do ouriço

Tabela 2: Testes efetuados com DSS, condições aplicadas, métodos estatísticos usados e resultados crônicos e agudos obtidos.

\begin{tabular}{cccc}
\hline Teste & Condição & CENO (Método) & CE $_{50}$ (Método) \\
& Temperatura/Duração & & \\
\hline \multirow{2}{*}{ Temperatura } & $20^{\circ} \mathrm{C} / 24 \mathrm{~h}$ & $0,37 \mathrm{mg} / \mathrm{l}$ & $1,49 \mathrm{mg} / \mathrm{l}$ \\
& & $($ Dunnetts $)$ & $($ Spearman) \\
& $25^{\circ} \mathrm{C} / 24 \mathrm{~h}$ & $0,75 \mathrm{mg} / \mathrm{l}$ & $1,88 \mathrm{mg} / \mathrm{l}$ \\
& & $($ Dunnetts $)$ & $($ Spearman) \\
Tempo de & $20^{\circ} \mathrm{C} / 24 \mathrm{~h}$ & $0,37 \mathrm{mg} / \mathrm{l}$ & $0,48 \mathrm{mg} / \mathrm{l}$ \\
Exposição & $20^{\circ} \mathrm{C} / 48 \mathrm{~h}$ & $($ Steels $)$ & $(\mathrm{Gráfico)}$ \\
& & $0,185 \mathrm{mg} / \mathrm{l}$ & $0,45 \mathrm{mg} / \mathrm{l}$ \\
& & $($ Steels $)$ & $($ Spearman) \\
\hline \hline
\end{tabular}


Arbacia punctulata $0 \mathrm{CE}_{50}$ foi de 7,03 e 5,43 $\mathrm{mg} / \mathrm{l}$, e para o anfípoda Ampelisca abdita ficou entre 5,16 e 9,56 mg/l. Estes dados comparativos exemplificam a maior sensibilidade de embriões de Perna perna frente a outros níveis tróficos, confirmando sua importância na utilização como organismo-teste em avaliações de impactos ambientais. A menor tolerância de organismos adultos de Perna perna em relação ao mexilhão Brachidontes solisianus (quando expostos a diferentes concentrações de um detergente aniônico) conforme demonstrado por Zuim \& Mendes (1980), também indica a fragilidade da espécie à perturbações ambientais.

Em estudo desenvolvido por Reis Fo. (1999) a aplicação de testes de toxicidade com água intersticial e elutriato de sedimentos provenientes do rio Itajaí-Açu (Itajaí) e Saco dos Limões (Baía Sul da llha de Santa Catarina Florianópolis) com embriões de Perna pernae Arbacia lixula (ouriço) foi marcante a alta sensibilidade apresentada pelos mexilhões em comparação aos ouriços.

\section{CONCLUSÕES}

-A técnica de indução de desovas através do sistema de fluxo contínuo com água do mar é a mais eficiente para Perna perna;

-Temperatura para o desenvolvimento de testes de toxicidade recomendado é de $20^{\circ} \mathrm{C}$;

-A salinidade de trabalho deve estar limitada entre 30 e $35 \%$;

-Para os tratamentos agudos $\left(\mathrm{CE}_{50}\right)$, os testes podem ser desenvolvidos em 24 horas, e para os crônicos (CENO), em 48 horas;

-Devido a sua alta sensibilidade, os percentuais de efeito observados nos frascos controle podem chegar a $30 \%$;

-O emprego do tratamento crônico deve ser priorizado em relação ao tratamento agudo;
- Os embriões de Perna perna possuem alta sensibilidade em comparação com outros organismos aplicados em testes crônicos marinhos.

\section{AGRADECIMETOS}

Os autores agradecem a Leonardo Rörig, Gilberto Manzoni e Valéria Bellotto pela revisão do manuscrito.

\section{REFERÊNCIAS BIBLIOGRÁFICAS}

Abessa, D.M.S.; Zaroni, L.P.; Rachid, B.R.F. \& E.C.P.M. Sousa. 1998. Sensibilidade de 4 organismos utilizados em testes de toxicidade ao dicromato de potássio e ao DSS. V Encontro Brasileiro de Ecotoxicologia (ECOTOX). Itajaí, Santa Catarina.(Anais). p. 72.

Amado Filho, G.M.; Karez, C.S.; Andrade, L. \& W.C. Pfeiffer. 1997. Zinc and Cadmium accumulation by Padina gymnospora (Phaeophyceae) from Sepetiba Bay (Rio de Janeiro, Brazil) over the last 8 years. VII Congresso Latino-Americano sobre Ciências do Mar (COLACMAR). Santos, SP. (Anais). p. 28-30.

ASTM. 1992. Standard guide for conducting static acute toxicity tests starting with embryos of four species of saltwater bivalve molluscs. E 724-89 p. 377-394.

Badaró-Pedroso, C. \& M.C.F. Santos. 1996a Avaliação preliminar dos efeitos da água de produção de petróleo sobre algumas espécies de organismos marinhos. III Simpósio sobre oceanografia. IOUSP, São Paulo. (Resumos). p. 310.

Badaró-Pedroso, C. \& M.C.F. Santos. 1996b. Utilização de larvas de Callinectes danae (Crustacea: Portunidae) em testes de toxicidade aguda. III Simpósio sobre oceanografia. IOUSP, São Paulo. (Resumos). p. 279. 
Badaró-Pedroso, C.; Santos, M.C.F. \& R.S. Carr. 1998. Utilization of a marine tie study to characterize toxicants in produced water samples. V Encontro Brasileiro de Ecotoxicologia (ECOTOX). Itajaí, Santa Catarina.(Anais). p. 73.

Bayne, B. L. 1965. Growth and the delay of metamorphosis of the larvae of Mytilus edulis (L.). Ophelia 2: 1-47.

Bayne, B.L. 1976. Marine Mussels: Their Ecology and Physiology. International Biological Programme 10. Cambridge University Press. Cambridge. London. 505 pp.

Bayne, B.L.; Garbbott, P.A. \& J. Widdows. 1975. Some effects of stress in the adult on the eggs and larvae of Mytilus edulis L. J. Mar. Biol. Ass. U.K. 55: 675-689.

Carr, R.S.; Long, E.D.; Windom, H.L.; Chapman, D.C.; Thursby, G.; Sloane, G.M. \& D.A. Wolfe. 1996. Sediment quality assessment studies of Tampa Bay, Florida. Environmental Toxicology and Chemistry. 15: 1218-1231.

Carvalheira, L.V.; Perin, G.; Pesce, A. \& S. Manente. 1997. Sediment elutriate toxicity determination in selected areas of Guanabara Bay. VII Congresso Latino-Americano sobre Ciências do Mar (COLACMAR). Santos, SP. (Anais). p. 149150.

Cherr, G.N.; Mcgee, J.S. \& J.M. Shenker. 1990. Methods for assessing fertilization and embryonic/larval development in toxicity tests using the California mussel (Mytilus californianus). Environmental Toxicology and Chemistry. 9: 1137-1145.

Davis, H.C. 1961. Effects of some pesticides on eggs and larvae of oyster (Crassostrea virginica) and clams (Venus mercenaria). Commercial Fisheries Review. 23: 8-23.

EPA. 1991. Methods for measuring the acute toxicity of effluents and receiving waters to freshwater and marine organisms. $4^{\circ}$ Edition. EMSL .293pp. Cincinnati.

Fernandes, F.C. 1981. Aspéctos biológicos e ecológicos do mexilhão Perna perna (Linné,
1758) da região de Cabo Frio - Brasil. Teste de Doutorado. IOUSP, SP. 90pp.

Fichet, D.; Radenac, G. \& P. Miramand. 1998. Experimental studies of impacts of harbour sediments resuspension to marine invertebrates larvae: biovailability of $\mathrm{Cd}, \mathrm{Cu}$, $\mathrm{Pb}$ and $\mathrm{Zn}$ and toxicity. Marine Pollution Bulletin. 36: 509-518.

Greenberg, A.E.; Clesceri, L.S. \& A.D. Eaton. 1992. Standard Methods for the examination of water and wastewater. APHA/AWWA/WEF.

Henriques, M.B. 2001. Avaliação dos bancos naturais do mexilhão Perna perna (Linnaeus, 1758) na Baía de Santos, Estado de São Paulo. Tese de Mestrado. UNESP, Rio Claro, SP. 74pp.

His, E. \& R. Beiras. 1995. Monitoring fresh and brackish water quality around Shellfish farming areas with a bivalve embryo and larva simplified Biossay method. Oceanologica Acta 18: 591-595.

Hooker, S.H. 1997. Larval and postlarval development of the New Zealand pipi, Paphies australis (Bivalvia: Mesodesmatidae). Bull. Mar. Sc. 61:225240.

Hunt, J.W. \& B.S. Anderson. 1993. From rresearch to routine: a review of toxicity testing with marine molluscs. Environmental Toxicology and Risk Assessment. Standart Techinical Publication. 320-339p.

Iwata, K.S. 1950. Spawning of Mytilus edulis: discharge by electrical stimulation. Bulletin of the Japanese Society of Scientific Fisheries. 15: 443-446.

Iwata, K.S. 1951. Spawning of Mytilus edulis: discharge by KCL injection. Bulletin of the Japanese Society of Scientific Fisheries. 16: 393-394.

Jorge, R.A.D.L.V.C. \& G.S. Moreira. 1998. Uso de mexilhões (Perna perna) como bioindicadores: determinação da mortalidade após 24 e 48 horas de exposição ao benzeno. V Encontro Brasileiro de 
Ecotoxicologia (ECOTOX). Itajaí, Santa Catarina.(Anais). p. 82.

Lucero, N.M.; Scelzo, M.A.; Giangiobbe, M.A. \& V.J. Moreno. 1997. Toxicidad aguda de un crudo, un dispersante y una mezcla de ambos sobre el camaron Artemesia longinaris (Crustacea: Penaeidae). VII Congresso Latino-Americano sobre Ciências do Mar (COLACMAR). Santos, SP. (Anais). p.108-109.

Moraes, R.B.C.; Pfeiffer, W.C.; Guimarães, J.R.D.; Borges, E. \& A. Campos. 1996. Avaliação da toxicidade de sedimentos das Baías de Guanabara e Sepetiba - RJ. III Simpósio sobre oceanografia. IOUSP, São Paulo. (Resumos). p. 328.

Nipper, M.G.; Badaró-Pedroso, C.; José, V.F. \& S.L.R. Melo. 1993. Toxicity testing with coastal species of southeastern Brazil. Mysis and Copepods. Bull. Envirom. Contam. Toxicol. 51: 83-95.

Rand, G.M. \& S.R. Petrocelli. 1985. Fundamentals of aquatic toxicology. Taylor \& Francis. $666 \mathrm{p}$.

Reis Fo, R.W. 1999. Estudos preliminares envolvendo testes de toxicidade embrio-larvais com o mexilhão Perna perna (Linnaeus, 1758). Monografia. Univ. do Vale do Itajaí.

Reish, D.J. 1988. The use of toxicity testing in marine environmental research. In: Soule, F.D. \& G.S. Kleppel (eds). Marine Organisms as Indicators. Springer-Verlag. p. 231-245.

Resgalla Jr., C.; De La Rocha, C.L. \& F. Morelli. 1997. Testes sub-letais com Acartia lilljeborgi Giesbrecht, 1892: efeitos do cromo e chumbo sobre as taxas de filtração, produção de ovos e eclosão. VII Congresso Latino-Americano sobre Ciências do Mar (COLACMAR). Santos, SP. (Anais). p. 328-330.

Resgalla Jr., C; Morelli, F.; Rodrigues-Ribeiro, M. \& A. Brandelli. 1998. Reprodução, de- senvolvimento embrio-larval e testes preliminares de toxicidade de Parablennius pilicornis (Cuvier, 1829)(Pisces: Blennidae). Notas Téc. FACIMAR 2: 41-49.

Rios, E. 1994. Seashells of Brazil. Rio Grande, FURG. $492 \mathrm{pp}$.

Romero, S.M.B. 1980. Características comportamentais e morfológicas dos estágios larvais de Perna perna (Lamellibranchia: Mytilidae) obtidos em laboratório. Bol. Fis. Animal. IBUSP. 4:4552.

Sagara, J. 1958. Artificial discharge of reproductive elements of certain bivalves caused by treatment of sea water and by injection with $\mathrm{NH}_{4} \mathrm{OH}$. Bulletin of the Japanese society of Scientific Fisheries. 23: 505-510.

Sampaio, L.A. \& M.B. Tesser. 1997. Toxicidade aguda da amônia para juvenis de Pampo, Trachinotus marginatus (Teleostei Carangidae). VII Congresso Latino-Americano sobre Ciências do Mar (COLACMAR). Santos, SP. (Anais). p. 385-386.

Wojciechowiski Jr., E. 1997. Monitoramento do Índice de condição de Mexilhões Perna perna no Parque de Mitilicultura na Enseada de Armação do Itapocoroy - Penha, SC. Monografia, UNIVALI.

Zamboni, A.; De Villa, R.A.; Gama, A.M.S.; Asp, N.E.; Omachi, C.Y.; Pinheiro, F.M.R.; Salomon, P.S. \& A. Vanz. 1996. Efeito da temperatura na toxicidade aguda de cobre e zinco sobre diferentes estágios de vida de Metamysidopsis elongata atlantica (crustacea: Mysidacea). III Simpósio sobre oceanografia. IOUSP, São Paulo. (Resumos). p. 284.

Zuim, S.M.F. \& E.G. Mendes. 1980. Tolerância de dois mexilhões marinhos, Perna perna e Brachidontes solisianus, a diferentes concentrações de um detergente aniônico. Rev. Brasil. Biol. 40: 585-590. 